\title{
Solution Derived p-ZnO/n-Si Nanowire Heterojunctions for Photodetection
}

Ken C. Pradel ${ }^{\dagger}$, Wipakorn Jevasuwan ${ }^{\ddagger}$, Joko Suwardy ${ }^{\ddagger}$, Yoshio Bando ${ }^{\ddagger}$, Naoki Fukata ${ }^{\ddagger}{ }^{\star}$, Zhong Lin Wang ${ }^{\dagger, \ddagger, *}$

${ }^{\dagger}$ School of Materials Science and Engineering, Georgia Institute of Technology, Atlanta, GA 30332-0245, USA

${ }^{\ddagger}$ International Center for Materials Nanoarchitectonics, National Institute for Materials Science, 1-1 Namiki, Tsukuba, 305-0044, Japan

Corresponding authors: zlwang@gatech.edu; FUKATA.Naoki@nims.go.jp

\begin{abstract}
While there is considerable interest in zinc oxide nanomaterials for optoelectronics research, one weakness of the material is the difficulty in producing p-type zinc oxide. This can be attributed to a number of factors such as acceptor instability, donor compensation during growth, and the formation of deep acceptors. Recently, it was discovered that antimony is a stable p-type dopant in hydrothermally grown $\mathrm{ZnO}$ nanowires, and this method has been modified to produce ultralong nanowires and homojunction thin films. In order to broaden the applicability of this new material, it is important to investigate how it interacts with other semiconducting nanomaterials. In this work we demonstrate a solution grown $\mathrm{p}-\mathrm{ZnO} / \mathrm{n}-\mathrm{Si}$ nanowire heterojunction. After investigating its basic materials properties, we show its applicability as an photodetector with responsivity as high as $1.02 \mathrm{~A} / \mathrm{W}$ at $442 \mathrm{~nm}$, an order of magnitude higher than similar nanostructures based on n-type $\mathrm{ZnO}$ and p-type Si.
\end{abstract}




\section{Introduction}

Zinc oxide nanomaterials have been a mainstay in research on the next generation of electronics and optoelectronics.[1-3] Its numerous properties such as piezoelectricity, high exciton binding energy, and a wide direct bandgap, have all been investigated thoroughly for fields such as photovoltaics,[4-6] LEDs,[7-9] nanogenerators,[10-12] and piezotronics.[13-15] However, one weakness of this material is that it is notoriously difficult to dope p-type.[16] ZnO is naturally n-type due to the unintentional doping caused by point defects during growth such as dissolved hydrogen[17] or zinc[18] interstitials. Over the years, many have tried doping a variety of Group I and V elements to produce p-type ZnO but have had difficulties in reproducibility and stability.[19,20] For example, while nitrogen should theoretically be an ideal dopant due to its similar atomic radius to oxygen and electronic structure, first-principles calculations and experimental observations have shown that it forms a deep acceptor, so it cannot cause hole conductivity in ZnO.[21,22] Furthermore, during the growth process, it is possible that compensating donor defects will form, canceling out the acceptors.[23] All these factors compounded together has caused the very feasibility of p-type $\mathrm{ZnO}$ to become the subject of controversy.

In 2011, it was demonstrated that antimony makes for a stable acceptor dopant in $\mathrm{ZnO}$.[24] Sb doped $\mathrm{ZnO}$ nanowires grown through this method remained stable for over 18 months,[25] which remains completely unprecedented. Rather than forming a $S b_{Z n}-2 V_{Z n}$ acceptor complex as had been previously postulated,[26] inversion domain boundaries formed by the Sb during growth leading to Sb and O codoping. Furthermore, it was grown using the low temperature hydrothermal method, which has numerous advantages such as compatibility with flexible polymer substrates,[27] and scalability.[28] Since its discovery, this method has been reproduced and modified to produce ultra-long nanowires to investigate its piezotronic properties,[29] and densely packed films to produce thin-film homojunctions to demonstrate its effect on nanogenerator performance.[30]

While there is a novelty in a pure $\mathrm{ZnO}$ system, in order to demonstrate the commercial applicability of p-type $\mathrm{ZnO}$, it is also important to show how it can be integrated with other semiconductors to produce heterojunction structures. Silicon is an ideal material for this demonstration as it is a thoroughly researched and well understood material which is the basis for our modern electronics. While $\mathrm{ZnO} / \mathrm{Si}$ heterojunctions have been extensively investigated in 
the past,[31-33] it has been done so with the opposite doping, i.e. n-type $\mathrm{ZnO}$ and p-type Si. For the first time, a solution derived $\mathrm{p}-\mathrm{ZnO} / \mathrm{n}-\mathrm{Si}$ heterojunction structure was demonstrated. To increase the contact area between the two materials, Si nanowires formed through nanoimprint lithography were used as the base with $\mathrm{ZnO}$ grown off of it. After investigating the basic materials properties of this structure, its applicability as a photodetector was demonstrated.

\section{Experimental Section}

Silicon core nanowires were formed through nanoimprint lithography and the Bosch process using n-Si(100) substrates.[34] Si substrates were patterned with $30 \mathrm{~nm}$-thick $\mathrm{Cr}$ layer by UV-imprint lithography and lift-off processes. Then, $\mathrm{SF}_{6}$ plasma followed by $\mathrm{C}_{4} \mathrm{~F}_{8}$ plasma was applied for deep Si etching to form SiNW structure. The flow rates of $\mathrm{SF}_{6}$ and $\mathrm{C}_{4} \mathrm{~F}_{8}$ were both fixed at $35 \mathrm{sccm}$ with a chamber pressure of $0.75 \mathrm{~Pa}$.

$\mathrm{ZnO}$ nanowires were then grown using the hydrothermal method. First, the Si nanowire samples were briefly etched in hydrofluoric acid to remove the oxide layer, and a $100 \mathrm{~nm}$ layer of $\mathrm{ZnO}$ was sputtered onto the nanowires to act as a seed layer for subsequent growth. From there, a solution containing $25 \mathrm{mM}$ zinc nitrate, $12.5 \mathrm{mM}$ hexamethylenetetramine (HMTA), 0.7 $\mathrm{mM}$ sodium citrate, $0.5 \mathrm{M}$ ammonium hydroxide, and $0.25 \mathrm{mM}$ antimony glycolate dopant solution was prepared. All chemicals were purchased from Wako Chemicals and used without further purification. The preparation of the dopant solution is described in detail in previous literature.[24] The Si substrates were floated face down on the solution surface and put into an oven heated to $75^{\circ} \mathrm{C}$ for 1 hour.

\section{Results and Discussion}

From the SEM image in Figure 1a, we can see that the Si nanowires produced through the Bosch process are of uniform dimensions and periodicity. The wires produced had a diameter of $150 \mathrm{~nm}$, a length of $1.3 \mu \mathrm{m}$ and a pitch of $500 \mathrm{~nm}$. Following $\mathrm{ZnO}$ nanowire growth, from the top view SEM image in Figure 1b, we can see urchin like $\mathrm{ZnO}$ nanowire structures with the same periodicity as the base Si. From the photograph in the inset, it appears that the $\mathrm{ZnO}$ nanowires grew over the entire sample. By cleaving the sample and looking at the cross section (Figure 1c), we see that the urchin structures form from the tips of the $\mathrm{Si}$ nanowires, where the $\mathrm{ZnO}$ nanowires fanned out during growth. Along the sides of the $\mathrm{Si}$ wires, the $\mathrm{ZnO}$ nanowires grew perpendicularly to the surface, coalescing and completely filling the spaces between $\mathrm{Si}$ nanowires. Sodium citrate has been shown to inhibit growth of $\mathrm{ZnO}$ nanowires along its c-axis, 
leading to shorter, but wider nanowires.[30,35] This allowed for the high degree of backfilling seen here. The length of the $\mathrm{ZnO}$ wires were limited by the growth of other $\mathrm{ZnO}$ on adjacent $\mathrm{Si}$ nanowires. The growth rate appears to have been uniform as the $\mathrm{ZnO}$ nanowires meet in the center. We can also observe a small amount of vertical growth from the bottom of the substrate, but the length is limited by the horizontal growth.

X-ray diffraction was used to further characterize the crystallinity of the as-grown structure (Figure 2a). As a control, first the Si nanowire array before $\mathrm{ZnO}$ growth was measured, and only a $\mathrm{Si}(400)$ peak is observed. As a comparison, the diffraction pattern of a $\mathrm{ZnO}$ nanowire array grown using the same solution on an unpatterned silicon wafer (Figure 2b) was also taken. As can be seen in the SEM image, due to the presence of sodium citrate, these nanowires grow into a densely packed film. The citrate ions bind to the (0001) surface, slowing down growth along the c-axis, thus producing shorter, but fatter wires, causing them to coalesce.[30,35] Because the $\mathrm{Sb}$ dopant interferes with normal $\mathrm{ZnO}$ growth, the nanowires also exhibit some roughness and irregularity. For this film, a $\mathrm{Si}(400)$ peak is still observed due to the underlying substrate, but several characteristic peaks for $\mathrm{ZnO}$ are also observed, the most prominent being the (002) peak. Because these densely packed nanowires are well aligned, a strong (002) peak is observed. The next strongest peaks at (103) and (101) correspond to the incompletely grown nanowire faces.[36] These correspond to the pyramidal faces in the inset of Figure 2a, which manifest during growth before the nanowire's equilibrium surfaces fully develop. There is always some irregularity during nanowire growth, but based on its relative intensity compared to the (002) peak, and the SEM image, this effect is minimal.

When taking the diffraction scan for the heterojunction structure, a combination of all the previously observed peaks are observed, in addition to the noticeable ZnO (100) and (110) peaks which correspond to the nonpolar side surfaces of the $\mathrm{ZnO}$ nanowire as shown in the schematic inset in Figure 2a. These peaks are only observed in this case due to the $\mathrm{ZnO}$ nanowires growing off the sides of the Si nanowire peaks, i.e. parallel to the substrate. Because the $\mathrm{ZnO}$ layer is no longer as thick in this sample compared to the film, $\mathrm{ZnO}(002)$ peak has become weaker relative to the $\mathrm{Si}(400)$ peak. Furthermore, the intensity of the (101) peak has also increased due to the larger number of incompletely grown nanowires. The $\mathrm{ZnO}$ grown on the $\mathrm{Si}$ nanowires have a tapered end as opposed to the complete hexagonal structure observed in the film sample. While in principle, the hexagonal wires observed in Figure $2 \mathrm{~b}$ should be obtainable, despite being 
grown at the same conditions, only partially grown nanowires were obtained. As the nano patterned Si substrate has a higher surface area than the flat surface, it's possible that the growth kinetics changed, resulting in incompletely grown nanowires.

In order to measure the device's electrical properties, $100 \mathrm{~nm}$ of ITO was sputtered onto the $\mathrm{ZnO}$ film to serve as the top electrode, while a silver contact was deposited on the back side of the Si wafer to form Ohmic contacts. Gold wires were attached to the substrate using silver paste as electrical leads. The completed structure is shown schematically in Figure 3. A Keithley 4200 semiconductor parameter analyzer was used to perform all electrical measurements. When the IV behavior was measured without any illumination under positive forward bias, rectification behavior is observed as seen by the black curve in Figure 3, showing that a $p$ - $n$ junction had formed. The leakage current is orders of magnitude lower than the forward bias current, showing that a good p-n junction formation between the two materials.

To test the homojunction's photoresponse, a laser with a wavelength of $442 \mathrm{~nm}$ was illuminated on the device. A continuous neutral density filter wheel was used to adjust the intensity of the incident laser (Figure 3). As the laser intensity increased from $0.49 \mu \mathrm{W}$ (purple) to $1.2 \mathrm{~mW}$ (red), the photocurrent under reverse bias increased dramatically. As the device is fairly conductive under forward bias to begin with, the photocurrent produced is rather insignificant. As a result, all further photoresponse measurements were performed when the device was under a constant reverse bias of $-1 \mathrm{~V}$.

In order to characterize the device as a photodetector, the sample was periodically exposed to the laser on and off for 10 seconds at a time. This process was repeated at different intensities which is split into two plots, Figure 4a\&b for clarity. An abrupt change in current can be observed when the device is exposed to the laser, even at the lowest power setting used, as shown in the inset of Figure 4b. Furthermore, the device has a low capacitance as there is no nonlinear behavior observed upon exposing or blocking the light source. This is made clear when zooming in on the moment of light exposure, where we can see that the current remains approximately constant just before and after the device is illuminated (Figure 4c). Furthermore, we can see that the photoresponse time was quite fast, less than $10 \mathrm{~ms}$, which is the detection limit of our equipment. 
In order to get a better idea of the photoresponse, quantitative analysis is necessary. A linear increase in current can be observed as the laser power increased. The standard method to normalize photoresponse for different power levels is a responsivity term $\mathrm{R}$ [37] defined as:

$$
R=\frac{I_{\text {light }}-I_{\text {dark }}}{P_{\text {light }}}
$$

where $\mathrm{I}_{\text {light }}$ and $\mathrm{I}_{\text {dark }}$ are the current under illumination and in the dark, respectively, and $\mathrm{P}_{\text {light }}$ is the power of the light source. The units for $\mathrm{R}$ are $\mathrm{A} / \mathrm{W}$. The average photoresponsivity at these intensities was $1.02 \mathrm{~A} / \mathrm{W}$ with a standard deviation of 0.07 . This is several orders of magnitude higher than the value observed for similar nanowire-nanowire heterojunction structures $(0.012)$ $\mathrm{A} / \mathrm{W})[31]$. This value is also several times higher than those based on $\mathrm{ZnO}$ nanowires and planar silicon (0.38)[38] and Si nanowires with hydrothermally grown ZnO nanoparticles (0.54)[39]. Due to the dense nanowire growth, there is a larger junction area, and thus more electron-hole pair generation.

\section{Conclusion}

In conclusion we have demonstrated the first $\mathrm{ZnO} / \mathrm{Si}$ nanostructured heterojunction based off of solution grown p-type $\mathrm{ZnO}$. The synthesis methods for both materials are simple and easily scalable, making this technique suitable for mass production. By using a solution designed to grow densely packed $\mathrm{ZnO}$ nanowires, it's possible to completely backfill the gaps between the $\mathrm{Si}$ nanowires, increasing the active area for photoresponse. The formation of a p-n junction between the two materials was confirmed, and a peak photoresponsivity of $1.02 \mathrm{~A} / \mathrm{W}$ was measured with a $442 \mathrm{~nm}$ light source. As Sb doped p-type $\mathrm{ZnO}$ is still a relatively new system, it is important to investigate its compatibility with other material systems in order to broaden its applications.

Acknowledgements. Research was supported by the National Science Foundation (DMR1505319), MANA, National Institute For Materials Science, Japan, and the NSF East Asia Pacific Summer Institutes (EAPSI) program. 


\section{Figures}
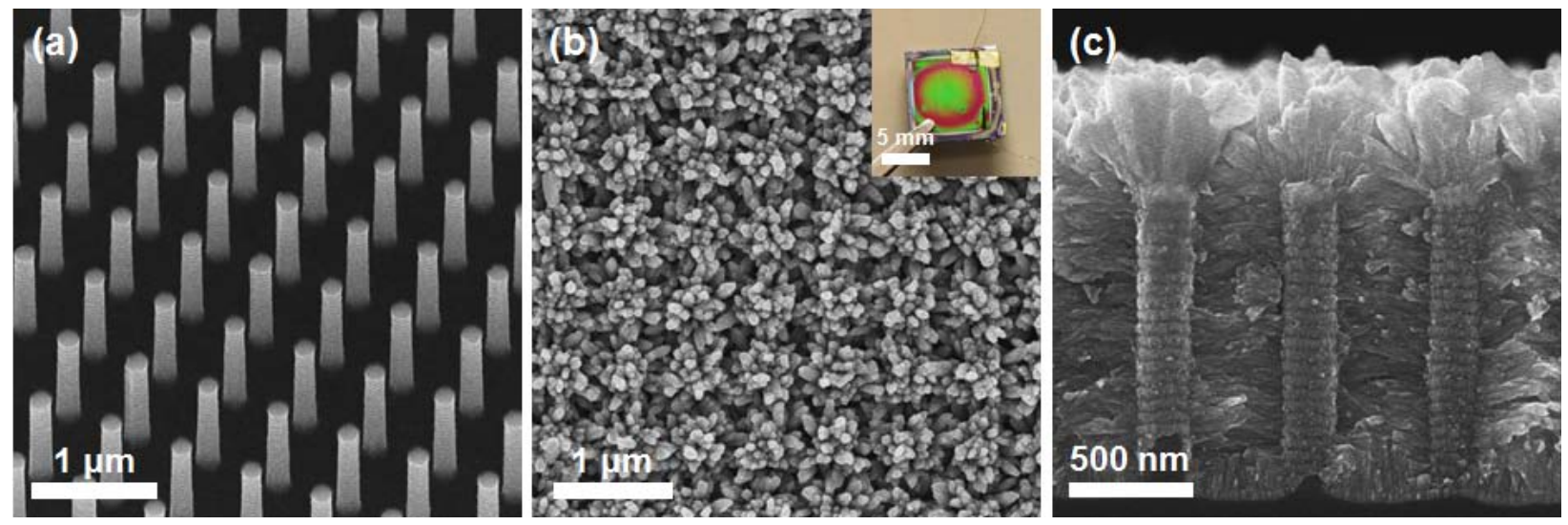

Figure 1. (a) SEM image of n-type Si nanowires grown using nanoimprint lithography.

Nanowires with diameter $150 \mathrm{~nm}$, length $1.3 \mu \mathrm{m}$, and pitch $500 \mathrm{~nm}$, are uniformly produced. (b) top and (c) side view SEM images of the Si nanowire array following p-type $\mathrm{ZnO}$ growth. The inset in (b) shows a photograph of the as grown heterojunction.
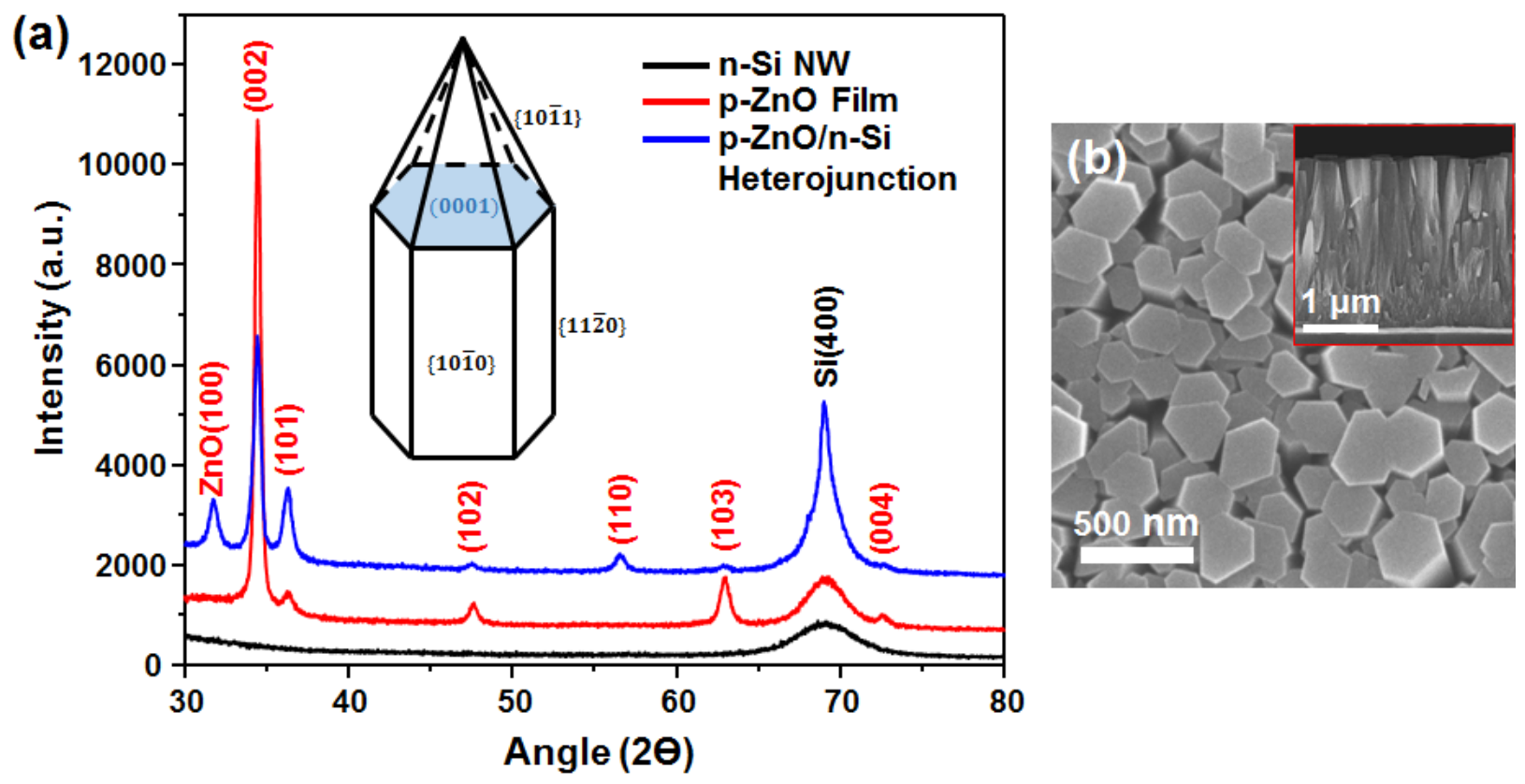

Figure 2. (a) XRD scans of the Si nanowire array before growth, a p-type ZnO film on a smooth Si substrate, and the n-Si/p-ZnO heterojunction. (b) SEM image of a p-type ZnO film grown on a smooth Si substrate. The inset shows a cross section of the same sample. 


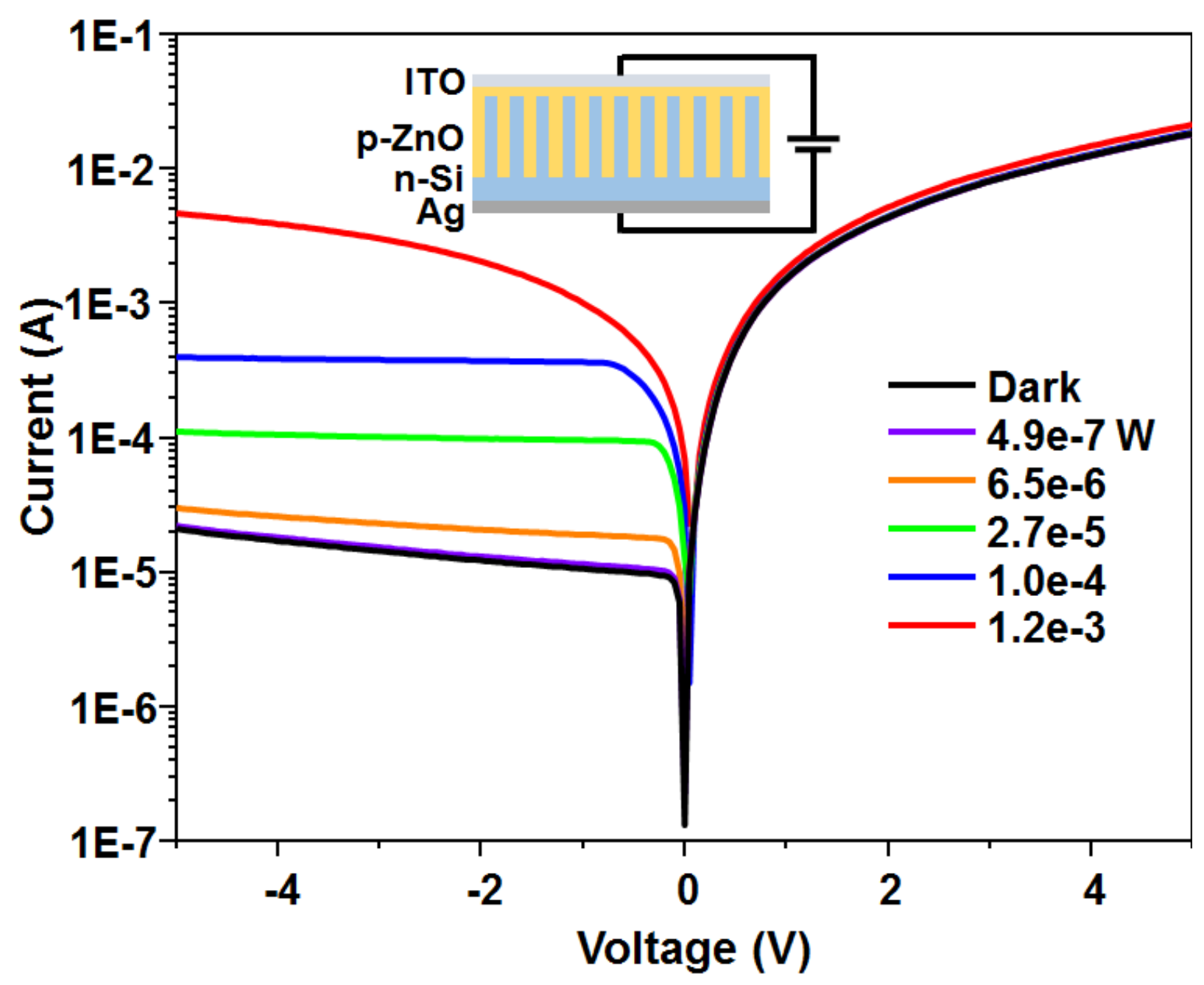

Figure 3. I-V behavior of the $\mathrm{p}-\mathrm{ZnO} / \mathrm{n}-\mathrm{Si}$ heterojunction structure. Measurements were taken in the dark and under illumination by a $442 \mathrm{~nm}$ laser under different power levels. The inset shows a schematic of the device structure. Under forward bias, there is not a strong photoresponse, while a noticeable response is observed under reverse bias. 

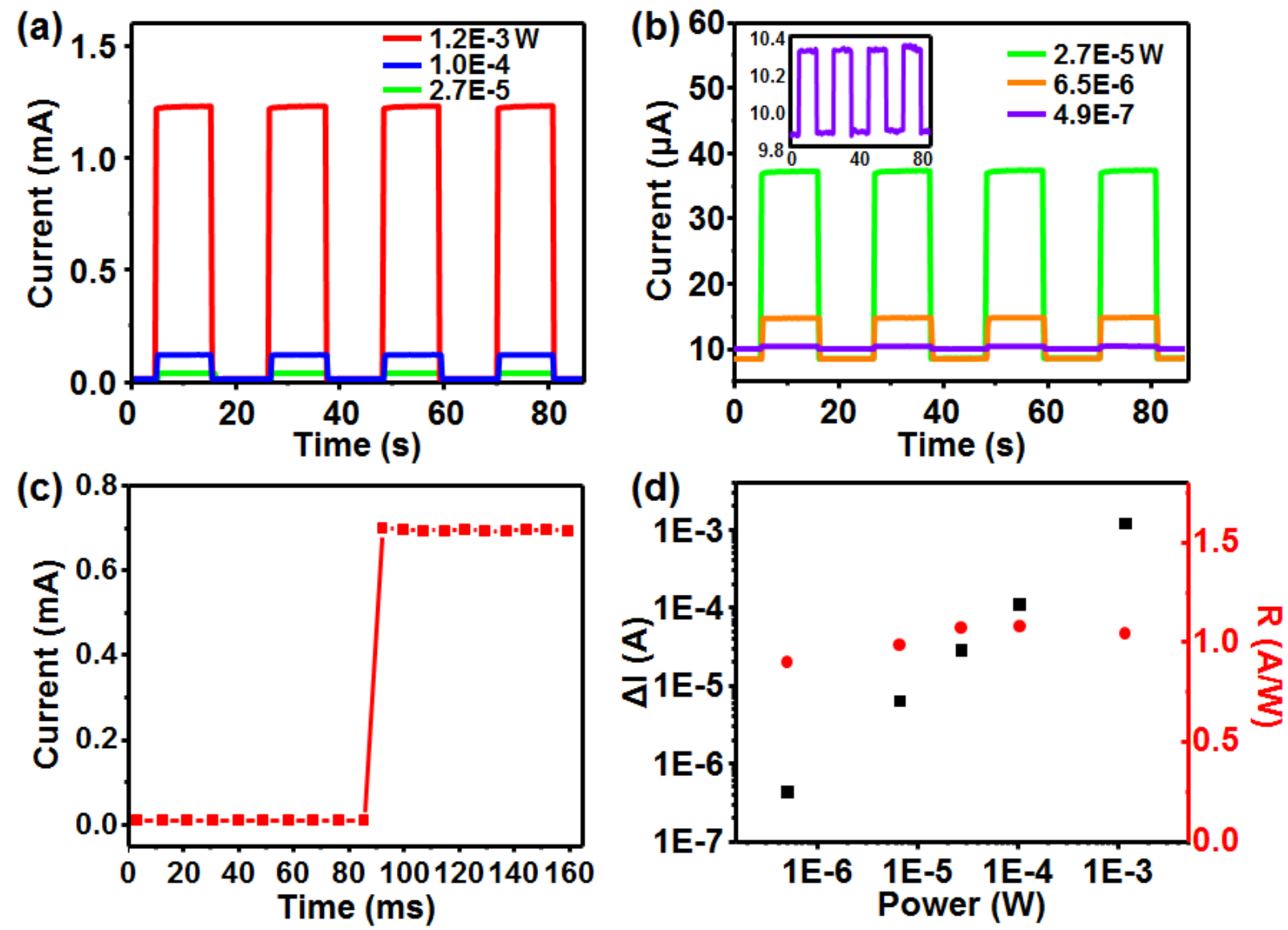

Figure 4. (a,b) Photocurrent response for heterojunction device to $442 \mathrm{~nm}$ laser at various power levels. The sample was exposed to the laser for 10 seconds, then blocked for 10 seconds in sequence. (c) Time response of the device to light exposure from the laser. The space between each point is $10 \mathrm{~ms}$. (d) Difference between light and dark current, and responsivity as a function of incident laser power. 


\section{References}

[1] Z.L. Wang, Materials today 7 (2004) 26.

[2] Z.L. Wang, Materials Science and Engineering: R: Reports 64 (2009) 33.

[3] A.B. Djurišić, A.M.C. Ng, X.Y. Chen, Progress in Quantum Electronics 34 (2010) 191.

[4] J.B. Baxter, E.S. Aydil, Applied Physics Letters 86 (2005) 053114.

[5] H. Jingbin, F. Fengru, X. Chen, L. Shisheng, W. Min, D. Xue, W. Zhong Lin, Nanotechnology 21 (2010) 405203.

[6] Y. Yang, W. Guo, Y. Zhang, Y. Ding, X. Wang, Z.L. Wang, Nano Letters 11 (2011) 4812.

[7] X.-M. Zhang, M.-Y. Lu, Y. Zhang, L.-J. Chen, Z.L. Wang, Advanced Materials 21 (2009) 2767.

[8] Q. Yang, W. Wang, S. Xu, Z.L. Wang, Nano Letters 11 (2011) 4012.

[9] C. Pan, L. Dong, G. Zhu, S. Niu, R. Yu, Q. Yang, Y. Liu, Z.L. Wang, Nature Photonics 7 (2013) 752.

[10] Z.L. Wang, J. Nanosci. Nanotechnol. 8 (2008) 27.

[11] Z.L. Wang, Mater. Sci. Eng. R-Rep. 64 (2009) 33.

[12] J. Briscoe, S. Dunn, Nano Energy 14 (2015) 15.

[13] Y. Zhang, Y. Liu, Z.L. Wang, Advanced Materials 23 (2011) 3004.

[14] W. Wu, X. Wen, Z.L. Wang, Science 340 (2013) 952.

[15] X. Wen, W. Wu, C. Pan, Y. Hu, Q. Yang, Z.L. Wang, Nano Energy (2014).

[16] C. Park, S. Zhang, S.-H. Wei, Physical Review B 66 (2002) 073202.

[17] C.G. Van de Walle, Physical Review Letters 85 (2000) 1012.

[18] S.B. Zhang, S.H. Wei, A. Zunger, Physical Review B 63 (2001) 075205.

[19] M.-P. Lu, M.-Y. Lu, L.-J. Chen, Nano Energy 1 (2012) 247.

[20] J.C. Fan, K.M. Sreekanth, Z. Xie, S.L. Chang, K.V. Rao, Progress in Materials Science 58 (2013) 874.

[21] J. Lyons, A. Janotti, C. Van de Walle, Applied Physics Letters 95 (2009) 252105.

[22] M.C. Tarun, M.Z. Iqbal, M.D. McCluskey, AIP Advances 1 (2011) 022105.

[23] W.-J. Lee, J. Kang, K. Chang, Physical Review B 73 (2006) 024117.

[24] F. Wang, J.-H. Seo, D. Bayerl, J. Shi, H. Mi, Z. Ma, D. Zhao, Y. Shuai, W. Zhou, X. Wang, Nanotechnology 22 (2011) 225602.

[25] A.B. Yankovich, B. Puchala, F. Wang, J.H. Seo, D. Morgan, X.D. Wang, Z.Q. Ma, A.V. Kvit, P.M. Voyles, Nano Letters 12 (2012) 1311.

[26] S. Limpijumnong, S.B. Zhang, S.-H. Wei, C.H. Park, Physical Review Letters 92 (2004) 155504.

[27] Y. Hu, Y. Zhang, C. Xu, L. Lin, R.L. Snyder, Z.L. Wang, Nano Letters 11 (2011) 2572.

[28] Y. Wei, W. Wu, R. Guo, D. Yuan, S. Das, Z.L. Wang, Nano Letters 10 (2010) 3414.

[29] K.C. Pradel, W.Z. Wu, Y.S. Zhou, X.N. Wen, Y. Ding, Z.L. Wang, Nano Letters 13 (2013) 2647.

[30] K.C. Pradel, W. Wu, Y. Ding, Z.L. Wang, Nano Letters 14 (2014) 6897.

[31] K. Sun, Y. Jing, N. Park, C. Li, Y. Bando, D. Wang, Journal of the American Chemical Society 132 (2010) 15465.

[32] H.-D. Um, S.A. Moiz, K.-T. Park, J.-Y. Jung, S.-W. Jee, C.H. Ahn, D.C. Kim, H.K. Cho, D.-W. Kim, J.-H. Lee, Applied Physics Letters 98 (2011) 033102.

[33] K. Sun, Y. Jing, C. Li, X. Zhang, R. Aguinaldo, A. Kargar, K. Madsen, K. Banu, Y. Zhou, Y. Bando, Z. Liu, D. Wang, Nanoscale 4 (2012) 1515. 
[34] K.J. Morton, G. Nieberg, S. Bai, S.Y. Chou, Nanotechnology 19 (2008) 345301.

[35] Z.R. Tian, J.A. Voigt, J. Liu, B. McKenzie, M.J. McDermott, M.A. Rodriguez, H. Konishi, H. Xu, Nat Mater 2 (2003) 821.

[36] J. Cheng, K.M. Poduska, ECS Journal of Solid State Science and Technology 3 (2014) P133.

[37] K.-J. Baeg, M. Binda, D. Natali, M. Caironi, Y.-Y. Noh, Advanced Materials 25 (2013) 4267.

[38] N.H. Al-Hardan, A. Jalar, M.A. Abdul Hamid, L.K. Keng, N.M. Ahmed, R. Shamsudin, Sensors and Actuators A: Physical 207 (2014) 61.

[39] D. Liu, X. Shen, T. Song, J. Hu, B. Sun, Physical Chemistry Chemical Physics 15 (2013) 4970. 

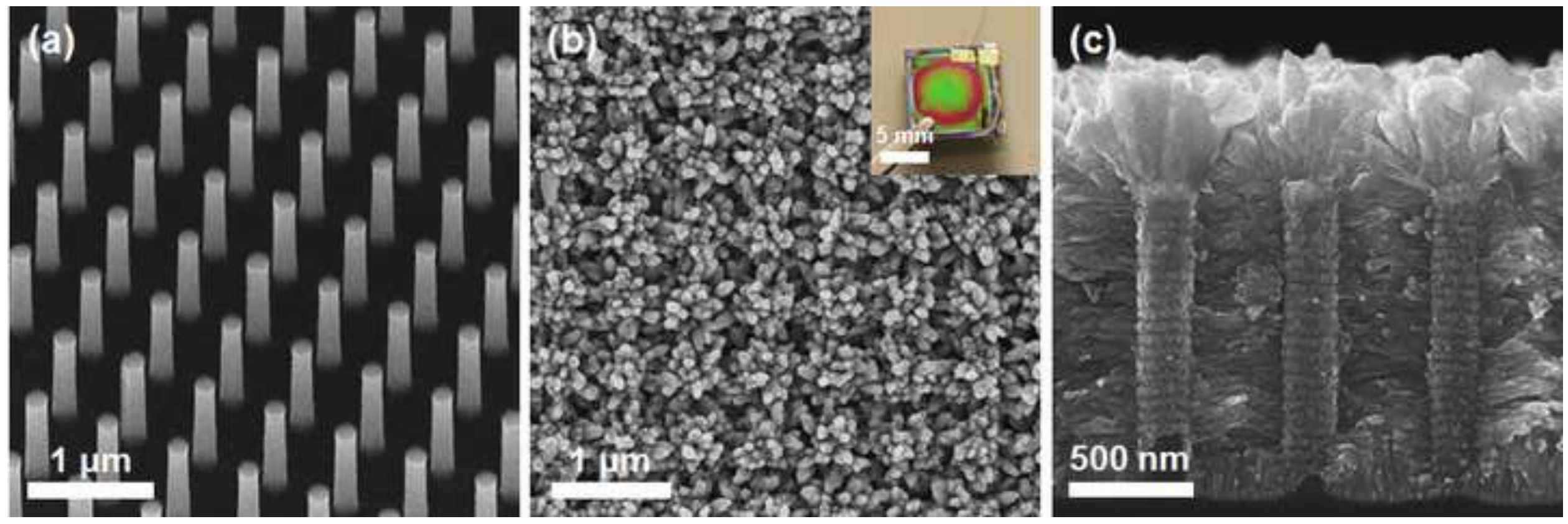